\title{
Classification of traumatic brain injury severity using retrospective data
}

\author{
Sandra Rogers *1, Amber W. Trickey ${ }^{2}$ \\ ${ }^{1}$ Malek School of Health Professions, Marymount University, Arlington County, Virginia, United States \\ ${ }^{2}$ Advanced Surgical Technology and Education Center (ASTEC), Inova Health System, Falls Church, Virginia, United States
}

Received: January 22, 2017

Accepted: June 8, 2017

Online Published: June 18, 2017

DOI: $10.5430 /$ jnep.v7n11p23

URL: https://doi.org/10.5430/jnep.v7n11p23

\begin{abstract}
Objective: Accurate classification of traumatic brain injury (TBI) severity is essential to brain injury research. TBI heterogeneity complicates classification of the injury; is a significant barrier in the design of therapeutic interventions; and results in retrospective data which is difficult to translate. The objective of this study is to describe the differences in two current tools used in the classification of TBI severity, the Glasgow Coma Scale (GCS) and the head Abbreviated Injury Score (AIS), using retrospective data to compare their performance.

Methods: Using correlational and descriptive statistics, this study examined two TBI severity classification methods across a large sample of TBI patients ( $\mathrm{N}=56,131)$, who were treated at level I and level II trauma centers in the United States and were

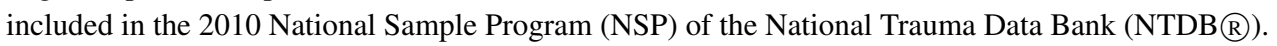

Results: The study population was $67 \%$ male, $67 \%$ non-Hispanic white, treated most often in trauma centers in the South (38\%), with blunt trauma (93\%) and from non-motor vehicle collisions (MVC's) (56\%). Observation of the AIS classification system demonstrated that it tends to over-score TBI severity compared to the GCS classification. The methods (GCS \& AIS) had a weak, inverse relationship with a correlation coefficient (Pearson's $r$ ) of -0.3980 , which was significant at $p<.001$.

Conclusions: The current study addressed the difficulties associated with categorizing TBI severity when analyzing retrospective data. Although AIS is commonly used to classify severity in retrospective data when GCS is unavailable, the relationship between the two scales is relatively unknown. Results show that AIS and GCS are more closely related for severely brain injured patients but in cases of mild and moderate injury, AIS is less predictive of GCS. Since they are often used in conjunction in identifying brain injured severity in retrospective data, researchers cannot be certain that the tools are similarly classifying mild, moderate, and severe injuries. This study reinforces the need for additional TBI severity classification methods, such as neuroimaging techniques and biomarkers.
\end{abstract}

Key Words: Traumatic brain injury, Moderate brain injury, Brain injury, GCS, AIS

\section{INTRODUCTION}

Accurate classification of traumatic brain injury (TBI) severity is essential to contemporary brain injury research and public health initiatives. The heterogeneity or diversity of impairments that patients suffer from is a relevant factor for planning, developing, and evaluating treatment and is related to the varied causes and pathophysiologic mechanisms responsible for the neurological deficits of patients. ${ }^{[1]}$ The heterogeneity of TBI leads to variation in the common classification of brain injuries as either mild, moderate, or

\footnotetext{
* Correspondence: Sandra Rogers; Email: srogers@ marymount.edu; Address: Malek School of Health Professions, Marymount University, Arlington County, Virginia, United States.
} 
severe and can also be a significant barrier in brain injury research. The classification of the brain injury is important because it often refers to the level of functioning immediately after a TBI and is discussed with regards to the severity of the brain injury; with patients typically divided into the broad categories of mild, moderate, and severe injury. ${ }^{[2]}$ Not only is treatment often based on these categories but also rehabilitation.

Over the past forty years, a variety of scoring systems that measure injury severity have been created and utilized in trauma care and research. These scoring systems are used by emergency medical services (EMS) to assess injury severity in the field triage so that the patient's level of care needs can be determined. ${ }^{[3]}$ The importance of injury scoring systems cannot be underestimated since the information obtained is used not only in making clinical decisions for the patient but also to compare the quality of trauma care in trauma related outcome research and benchmarking. The accuracy and reliability of the TBI injury scoring tool and the fact that it indeed measures what it aims to measure are of upmost importance. ${ }^{[3]}$

The two most commonly used methods of designating the brain trauma severity after an injury are the Glasgow Coma Scale (GCS) and the head Abbreviated Injury Score (AIS) classification tools. ${ }^{[2]}$ The GCS is a physiologic scale based on clinical examination and the AIS head score is an anatomic score based on computerized tomography (CT) scan, operative or autopsy findings. ${ }^{[4]}$ Although GCS is the most widely used method to classify brain injury in the clinical setting, a major difficulty in retrospective research is that the GCS variable is often missing in a large percentage of patients in large database cohorts. This results in the use of other measures such as the head AIS to classify mild, moderate, or severe injury, even though the head AIS scale does not have established number ranges for these classifications. ${ }^{[5]}$ The missing GCS score in the clinical setting is often related to the lack of training and nurses' lack of confidence in GCS proficiency. ${ }^{[6]}$ It is imperative to have consistent methods of TBI severity stratification.

Retrospective data are essential in studying TBI. Trauma databases are increasingly being used to formulate predictive models in trauma, as well as clinical documentation, prognostication, and TBI research. ${ }^{[7]}$ An important strength of most retrospective databases is that they allow researchers to examine medical care utilization as it occurred in routine clinical care. Retrospective databases often provide large study populations and longer observational periods, allowing for examination of specific subpopulations. In addition, retrospective databases provide a relatively inexpensive and expedient approach for answering the time-sensitive questions posed by decision makers. ${ }^{[8]}$

Therefore, the primary purpose of this paper is to describe TBI severity classification in a large sample of patients using retrospective data and to compare the GCS and the AIS head score performance and evaluate the differences. Secondary objectives were to compare demographic and clinical characteristics of TBI patients classified using the two methods: GCS and AIS of the head.

\section{Methods}

\subsection{Data source}

TBI patient data were extracted from the 2010 National Sample Project (NSP) of the National Trauma Data Bank (NTDB $R$ ) database. The NTDB $\AA$ contains over 3 million records submitted voluntarily from over 900 United States trauma centers. Maintained by the American College of Surgeons (ACS), the registry contains information on patient demographics, payment source, injury type and severity, hospital treatment, and facility characteristics including teaching status, trauma center level, and bed size. ${ }^{[9]}$ The NTDB $R$ is the largest trauma registry in the U.S. and, owing to the large number of subjects and trauma centers, researchers are increasingly using this databank to explore different aspects of injury epidemiology and trauma care. ${ }^{[10]}$

The 2010 National Sample Project (NSP) is sampled from the NTDB $\cap$ and uses a stratified sample design of 100 designated sample hospitals in their sample frame and uses number of Emergency Department (ED) visits from the American Hospital Association 2005 data as the size measure. The strata used for the sampling include NTDB $R$ contributing

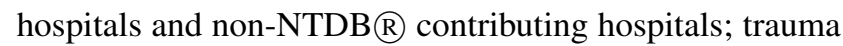
centers level I or II; and region, including Northeast, Midwest, West, and South. The NSP includes incident-level records and hospital information such as admission and discharge status, patient demographics, injury and diagnosis, procedure codes, injury severity scores, outcome variables, and weights [9].

\subsection{Study population}

A retrospective sample $(\mathrm{N}=56,131)$ of de-identified subjects from the 2010 National Sample Project (NSP) dataset who were diagnosed with a TBI based on the International Classification of Disease 9th Revision Clinical Modification (ICD-9-CM) codes were selected for the study. Based on the World Health Organization's Ninth Revision, the International Classification of Diseases, Clinical Modification (ICD-9-CM) is the official system used to assign codes to diagnoses and procedures associated with hospital utilization in the United States. Subjects for this study had at least 1 
ICD-9-CM code that met the CDC case definition for TBI, including codes 800.0-801.9, 803.0-804.9, 850.0-854.1, and 959.01. ${ }^{[11]}$

\subsection{Variables of interest}

Glasgow Coma Scale and Abbreviated Injury Scale Score of the head were selected because of their widespread use in classifying TBI severity in the literature. Both are common measures used to define TBI severity as mild, moderate or severe and have been used in both prospective and retrospective research. The GCS score ranges from 1 to 15 , with a severe brain injury falling between the scores of 3 and 8; moderate (9-12 score); and mild (13-15 score). The AIS of the head score ranges from a score of 1-6. For purposes of this study and based on prior use of the tool in brain injury research; the head AIS score was categorized as severe (5-6); moderate (3-4); and mild (1-2). The AIS of the head scores were grouped per cross-linkage of injury registry information in prior research. ${ }^{[12]}$

The following demographic and clinical data elements were retrieved from the NSP for analysis: age, sex, race/ethnicity, region of care, injury severity score (ISS), mechanism, trauma type, and mortality. Age was analyzed as a categorical variable; gender coded as male/female; and race/ethnicity was categorized as Hispanic/Latino, non-Hispanic White, non-Hispanic Black, and non-Hispanic other. Region included the standard classifications provided by the NSP of Midwest, Northeast, South, and West. ISS was categorized per the literature as ISS $<9,9-15,16-24$, and $\geq 25$. ISS ranges from 1-75 with a higher number associated with a more severe injury. The ISS score is calculated by taking the highest AIS score for each body region, squaring each, and then the addition of the three highest numbers. ${ }^{[3]}$ Mechanism of injury was either motor-vehicle collision (MVC) or non-MVC with trauma type listed as blunt or penetrating. Percentage of deaths was defined as in-hospital mortality.

\subsection{TBI severity}

TBI can be characterized as acute disruption of brain physiology or anatomic damage caused by an external source. The severity of the brain injury can be conveyed through injury severity classification. ${ }^{[13]}$ Generally, studies of the brain have classified patients based on the severity of the brain injury, and not by the associated extracranial injuries. ${ }^{[2]}$ Mild traumatic brain injuries have routinely been defined as a GCS score of 13 to 15 in the TBI literature. Moderate injures relate to a GCS score of 9 to 12; and a GCS score of 3 to 8 is associated with severe brain injuries. The GCS scores are based on level of consciousness, regardless of CT scan findings. ${ }^{[2]}$

Published by Sciedu Press

\subsection{Abbreviated Injury Scale score of the head}

The American Medical Association Committee on Medical Aspects of Automotive Safety initially developed the Abbreviated Injury Scale (AIS) for researchers to have a consistent method of comparing tissue damage injuries acquired in automobile crashes as well as having standardized language and classification of injuries. ${ }^{[14]}$ The AIS assigns a severity score to an injury on a scale from 1 (minor) to 6 (major), where a score of 1 generally does not require inpatient hospital treatment and a score of 6 is almost certainly fatal. ${ }^{[15]}$ Known as the gold standard in injury severity scoring since its development in 1971, the AIS is the foundation subsequent injury scoring tools, such as the Injury Severity Score (ISS). There have been several revisions of the AIS since its development. The AIS classifies the severity of injury in each region including the head, face, neck, thorax, abdomen, spine, upper and lower extremities and external/other. Unlike the GCS score, the AIS score cannot be calculated by hand at the scene of the trauma and requires manual review of the patient's record. This contributes to GCS generally being the first classification of brain injury by prehospital personnel or upon admission to acute care. The development of AIS software has made it possible to convert ICD-9-CM diagnosis to AIS codes by using a 7-digit number that describes type, location, and severity of the injury. ${ }^{[15]}$

A 2005 revision of the AIS (AIS-2005) changed the head section to be more descriptive and to include details of head injuries, such as the size of a hematoma. This change gave a better reflection of the clinical severity of the head injury and improved precision in the coding of the concussive head injury in a trauma registry dataset. ${ }^{[16]}$ In general, the head AIS measures and describes head injury severity based on a combination of symptoms (e.g. headache, amnesia, loss of consciousness), physical and neurologic examination findings (e.g. motor responses), and radiographic or CT scan findings (e.g. types of fracture, number, location, types, and volume of hemorrhage). ${ }^{[7]}$

Based on the initial CT scan, the AIS score has been shown to provide useful prognostic information in patients with severe head injury. A good or moderate outcome on the GOS was strongly predicted by an AIS of 3 or less (positive predictive value $95 \%$, specificity $98 \%$, sensitivity $40 \%$ ). However, the AIS was less predictive with a score of 5 (positive predictive value $71 \%$, specificity $75 \%$, sensitivity $67 \%) .{ }^{[17]}$

\subsection{Glasgow Coma Scale}

Developed to quantify the level of consciousness, the Glasgow Coma Scale has become a medical classic. ${ }^{[18]}$ In the past 30 years since its development, many coma scales have been recommended as alternatives to the GCS, but none 
have achieved success. With more than 4,500 publications referencing use of the GCS and by virtue of its simplicity, the scale has become the most commonly used and validated consciousness scale throughout the world. ${ }^{[19]}$ The GCS score has been shown to be particularly beneficial in the clinical management and prognosis of TBI, however, it does not offer specifics on the pathophysiology mechanisms that account for the neurological deficits present. ${ }^{[20]}$

Pre-hospital and emergency care personnel have widely used the GCS score to support the triage of trauma patients. ${ }^{[3]}$ The GCS scale is an objective scale that has three elements including eyes, verbal, and motor. Each element has a range of values: eyes (1-4), verbal (1-5), and motor (1-6). The GCS is the summation of these elements and is generally separated into three classifications; severe $(\leq 8)$, moderate (9-12), and minor ( $\geq 13)$. With an intra-class correlation coefficient of 0.8 to 1.0 for proficient users, a Cronbach's alpha of 0.69 , and a test-retest Spearman rho of 0.85, the GCS has established reliability. Validity of the scale is established by its predictive value 0.56 (Pearson $r$ ) when used alone to forecast 3-month survival, and when used with age and brainstem reflexes, the scale has a sensitivity of $79 \%$ to $97 \%$ with a specificity of $84 \%$ to $97 \% .{ }^{[21]}$ The GCS scale has been shown to possess the necessary methodological discipline and the quality of measurements needed in medical research and clinical practice care of head trauma patients, if it is used by experienced or trained personnel. ${ }^{[22]}$ The scale remains an important neurological assessment measure after head injury. In the area of research, the classification of the head trauma severity is still based on the admission GCS, regardless of the findings of the head CT scan. The gold standard for head trauma patients' outcome prediction remains the GOS. ${ }^{[19]}$

\subsection{Outcomes}

GCS and AIS of the head have been used in prior research to classify TBI samples to examine outcomes of mortality, function, and discharge destination after acute care. Research examining discharge destination as an outcome after TBI shows a consistency in the use of GCS in classifying mild (13-15), moderate (9-12), and severe (3-8) injuries. However, variations of brain injury classification as mild, moderate, and severe with the head AIS scale have been used to classify severity in prior research utilizing retrospective data as demonstrated in Table 1. Although the head AIS score is often being used when the GCS score or classification is missing, it is not clear what head AIS score correlates with the GCS classification of mild, moderate, or severe.

Table 1. Use of GCS and AIS scores to classify TBI severity in the literature

\begin{tabular}{llll}
\hline Author & TBI Severity & Classification Criteria & Data Type \\
\hline Hoffman et al., 2012 & Mild, Moderate, and Severe & AIS of head $\geq 2$ & Retrospective \\
Mosenthal et al., 2002 & Mild, Moderate, and Severe & AIS of head $\geq 3$ & Retrospective \\
Cuthbert et al., 2011 & Moderate and Severe & GCS 3 to 12 AIS of head $\geq 3$ & Retrospective \\
Bowman et al., 2007 & Moderate and Severe & AIS of head $\geq 3$ & Retrospective \\
Chan et al., 2001 & Moderate and Severe & AIS of head $\geq 2$ & Retrospective \\
Mellick et al., 2003 & Severe & AIS of head $\geq 4$ & Retrospective \\
\hline
\end{tabular}

\subsection{Statistical analyses}

Descriptive statistics are reported, including means, modes, medians or counts and percentages, as appropriate. Injury severity variables and descriptive variables were categorized using clinically conventional definitions. Pearson's correlational analysis was used to compare the interrelationships of the injury severity variables, GCS and AIS score of the head. A $p$ value $<.05$ was considered statistically significant. Descriptive plots were generated to compare the distribution of TBI severity scores between the AIS and GCS scales. The difference in TBI severity scores between the two scales is presented, based on the Bland-Altman approach. ${ }^{[23]}$ All statistical analyses were conducted using the statistical software program Stata $\AA$ version 13 (College Station, TX).

\section{RESULTS}

Retrospective data from the 2010 NSP dataset was analyzed to examine TBI severity classification methods in a sample of patients $(\mathrm{N}=56,131)$ that received care at either level I or level II trauma centers in the United States. The study population was $67 \%$ male, $67 \%$ non-Hispanic white, mostly sustaining blunt trauma (93\%) as compared to penetrating trauma; and of the blunt trauma, 56\% were non-MVC related injuries. The in-hospital mortality rate was $6 \%$. Three percent of the sample was missing GCS scores and resulted in a total of $\mathrm{N}=54,621$ patients examined using GCS scores while $7 \%$ of the sample were missing AIS head scores for a total of $\mathrm{N}=52,105$ examined using the AIS of head scores. The relationship between GCS and AIS is apparent. The majority of patients with AIS 1-4 had mild GCS scores, 
while most patients with AIS 5-6 had severe GCS scores (see Figure 1).

Differences were also noted in age and mechanism characteristics when examined across GCS and AIS severity levels of mild, moderate, severe. The age group 18-24 sustained a higher percentage of severe GCS injuries (18\%) with a higher percentage of MVC related injuries (53\%). While $21 \%$ of the 25-34 age group sustained severe injuries according to AIS (5-6); $19 \%$ sustained mild injuries as categorized by AIS of $1 \& 2$. Unlike GCS, a higher percentage of MVC related injuries ( $46 \%$ \& 58\%) were captured in the AIS mild scores of $1 \& 2$ respectively. ISS scores were appropriate with AIS scores increasing with severity and GCS scores showing an appropriately inverse relationship, considering that a lower number on the GCS is considered a severe injury. The majority of the injuries were classified as mild (77\%) per GCS scores with a higher percentage classified as moderate (46\%) according to AIS scores. Region of care for the AIS of the head scores was slightly different than the GCS characteristics in that $50 \%$ of the patients classifying as AIS of 1 received treatment in the West.

To distinguish correlation between head AIS scores (1-6) and GCS (3-15), an analysis was performed. The correlation coefficient Pearson's $r$ was significant at $p<.001$, with a weak, inverse relationship of -0.3980 . The inverse relationship was expected due to the direction of scoring with lower GCS scores and higher AIS scores associated with increased severity.

Observations from the Bland-Altman analysis gave the appearance that an AIS classification system tends to over score TBI severity compared to the GCS classification (see Figure 2).

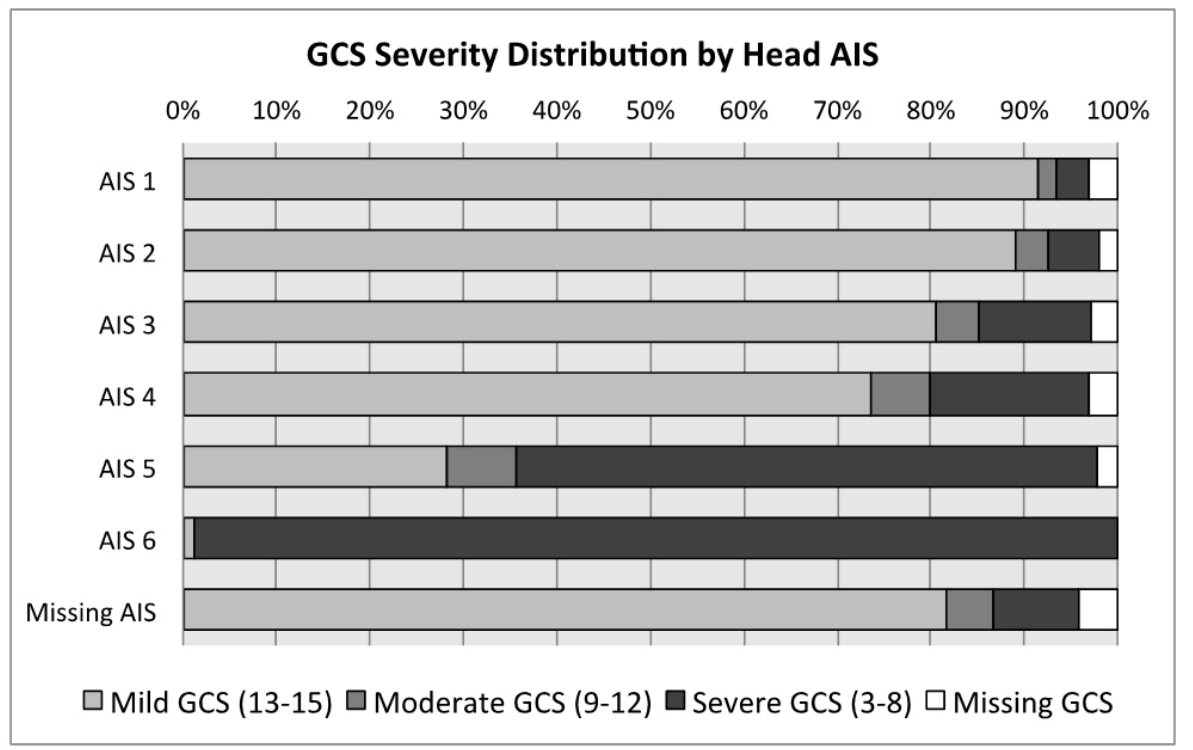

Figure 1. GCS severity distribution by head AIS

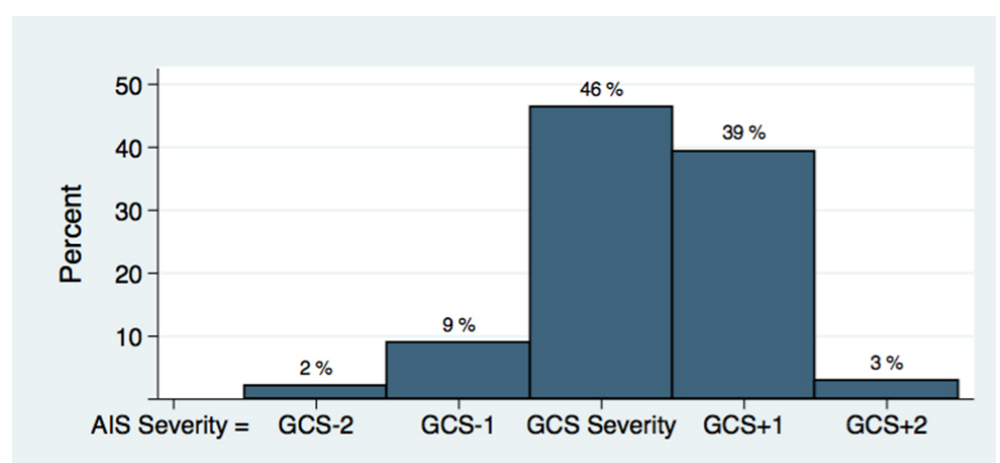

Figure 2. Plot of difference between AIS and GCS severity scales

Caption: Distribution of difference between AIS and GCS severity scales. Only 5\% of patients had a severe TBI by one scale and a mild TBI on the other scale (2\% severe GCS, $3 \%$ mild GCS). While $46 \%$ of patients had equivalent TBI severity by both scales, AIS scored one level higher in 39\%, and scored two levels higher for 3\% of patients - as evidenced on Figure 2 as GCS score +1 and GCS score +2 . 


\section{DISCUSSION AND CONCLUSION}

The classification of brain injury severity as either mild, moderate, or severe can result in different treatment modalities in the clinical setting. The GCS score is a quick and easy tool that is often used pre-hospital as well as upon admission to an acute care setting to classify the brain injured patient's injury as either mild, moderate, or severe. In retrospective research of TBI, the GCS classification is often used when isolating either the mild, moderate, and severe TBI patient populations. Missing GCS scores continue to plague retrospective data and are often substituted with the head AIS variable. However, there is variability in severity classification when using AIS head scores to stratify patients as mild, moderate, and severe. GCS remains the standard tool for TBI severity classification in prior literature with scores of 13-15 being labeled as mild; 9-12 as moderate; and a score between 3-8 classified as severe head injury. AIS of the head scores are often substituted when GCS is missing even though AIS of the head scores have not been routinely used for characterizing the severity of head injury in the literature. Prior researchers have used different levels of the AIS of the head score to match with the GCS rating of mild, moderate, and severe. The GCS and head AIS scales remain the most widely used methods to describe severity of head injury even though they may not necessarily be measuring the same features of traumatic brain injury, as evidenced by our correlation results.

This study offers an extensive examination of the two tools, GCS and AIS of the head, at different brain injury severity levels (mild, moderate, and severe) and highlights their similarities and differences. This study confirmed that AIS performs differently and demonstrated that AIS classification could be over scoring severity. Therefore, sensitivity analyses are recommended when stratifying severity based on a combination of methods, such as GCS and AIS head scores, to assess for any unexpected relationships and to reduce uncertainty.

Severity classification of brain injury (mild, moderate, severe) using tools with unclear correlation complicates future TBI research using retrospective data and the results. Injuries need to be coded consistently for comparison and for evaluation of trends. Researchers that use the NTDB and other large trauma datasets for evaluation of different aspects of injury epidemiology and trauma care should be aware of the special features and methodological challenges that may affect the robustness of their studies. With the inherent issues of missing data in retrospective databases, the use of multiple tools to stratify TBI severity is most often unavoidable. The results of this study provide feedback on how two common tools, GCS and AIS of head score, compare in TBI stratification for consideration in future TBI research. Although the GCS tool is commonly used by nurses in the clinical and prehospital setting, the AIS score may not be as common and could require the need for nursing education on the use of the tool in the clinical setting.

\section{ACKNOWLEDGEMENTS}

Committee on Trauma, American College of Surgeons, NTDB NSP 2010, Chicago, IL. The content reproduced from the NTDB NSP remains the full and exclusive copyrighted property of the American College of Surgeons. The American College of Surgeons is not responsible for any claims arising from works based on the original Data, Text, Tables, or Figures.

\section{CONFlicts OF INTEREST Disclosure}

The author declares that there is no conflict of interest.

\section{REFERENCES}

[1] Shapii A, Mat Zin N, Elaklouk AM. A game system for cognitive rehabilitation. Biomed Research International. 2014. 1-7 p.

[2] Stead LG, et al. TBI surveillance using the common data elements for traumatic brain injury: a population study. International Journal of Emergency Medicine. 2013; 6(5): 1-7. https ://doi .org/10.1 186/1865-1380-6-5

[3] Kim YJ. Injury severity scoring systems: a review of application to practice. Nursing in Critical Care. 2012; 17(3): 138-150.

[4] Demetriades D. Mortality prediction of head abbreviated injury score and Glasgow Coma Scale: analysis of 7,764 head injuries1. Journal of the American College of Surgeons. 2004; 199(2): 216-222. https ://doi.org/10.1016/j.jamcollsurg. 2004.02.030

[5] Cuff S, DiRusso S, Sullivan T, et al. Validation of a Relative Head Injury Severity Scale for Pediatric Trauma: The Journal of
Trauma: Injury, Infection, and Critical Care. 2014; 63(1): 172-178. https://doi.org/10.1097/TA.0b013e31805c14b1

[6] Mattar I, Liaw SY, Chan MF. Nurses' self-confidence and attitudes in using the Glasgow Coma Scale: a primary study. British Association of Critical Care Nurses. 2014; 20(2): 98-107. PMid:24450732 https://doi.org/10.1111/nicc. 12077

[7] Timmons SD, Bee T, Webb S, et al. Using the Abbreviated Injury Severity and Glasgow Coma Scale Scores to Predict 2-Week Mortality After Traumatic Brain Injury: The Journal of Trauma: Injury, Infection, and Critical Care. 2011; 71(5): 1172-1178. https : //doi.org/10.1097/TA.0b013e31822b0f4b

[8] Motheral B, Brooks J, Clark MA, et al. A Checklist for Retrospective Database Studies-Report of the ISPOR Task Force on Retrospective Databases. Value in Health. 2003; 6(2): 90-97.

[9] ACS. Resources for Optimal Care of the Injured Patient: 2006 (1 
edition.). American College of Surgeons. 2007.

[10] Roudsari B, Field C, Caetano R. Clustered and missing data in the US National Trauma Data Bank: implications for analysis. Injury Prevention. 2008; 14(2): 96-100. https://doi.org/10.1136/ip .2007 .017129

[11] Medicare C, Baltimore MS, Usa M. Home. 2013. Available from: http://www.cms.gov/

[12] Olson CJ, Brand D, Mullins RJ, et al. Time to death of hospitalized injured patients as a measure of quality of care. The Journal of Trauma. 2003; 55(1): 45-52. https://doi .org/10.1097/01.TA.000007 1291.17287 .57

[13] Menon DK, Schwab K, Wright DW, et al. Position Statement: Definition of Traumatic Brain Injury. Archives of Physical Medicine and Rehabilitation. 2010; 91(11): 1637-1640. https://doi.org/10.1 016/j.apmr.2010.05.017

[14] Linn S. The injury severity score-Importance and uses. Annals of Epidemiology. 1995; 5(6): 440-446. https ://doi.org/10.1016/ 1047-2797 (95) 00059-3

[15] Stephenson SCR, Langley JD, Civil ID. Comparing Measures of Injury Severity for Use with Large Databases. Journal of Trauma-Injury Infection. 2002; 53(2): 326-332. https ://doi .org/10.1097/00 005373-200208000-00023

[16] Carroll CP, Cochran JA, Price JP, et al. The AIS-2005 Revision in Severe Traumatic Brain Injury: Mission Accomplished or Problems for Future Research? Annals of Advances in Automotive Medicine / Annual Scientific Conference. 2010; 54: 233-238.

[17] Walder AD, Yeoman PM, Turnbull A. The abbreviated injury scale as a predictor of outcome of severe head injury. Intensive Care Medicine.
1995; 21(7): 606-609. PMid:7593906 https://doi.org/10.100 7/BF01700170

[18] Teasdale G, Jennett B. Assessment of coma and impaired consciousness. A practical scale. Lancet. 1974; 2(7872): 81-84. https: //doi.org/10.1016/S0140-6736(74)91639-0

[19] Balestreri M, Czosnyka M, Chatfield DA, et al. Predictive value of Glasgow coma scale after brain trauma: change in trend over the past ten years. J Neurol Neurosurg Psychiatry. 2004; 75: 161-162. PMid:14707332

[20] Saatman KE, Duhaime AC, Bullock R, et al. Classification of Traumatic Brain Injury for Targeted Therapies. Journal of Neurotrauma. 2008; 25(7): 719-738. https ://doi .org/10.1089/neu.2008.0 586

[21] Prasad K. The Glasgow coma scale: A critical appraisal of its clinimetric properties. Journal of Clinical Epidemiology. 1996; 49(7): 755-763. https://doi .org/10.1016/0895-4356(96)0 0013-3

[22] Stanczak DE, White JG, Gouview WD, et al. Assessment of level of consciousness following severe neurological insult. A comparison of the psychometric qualities of the Glasgow Coma Scale and the Comprehensive Level of Consciousness Scale. Journal of Neurosurgery. 1984; 60(5): 955-960. https ://doi.org/10.3171/jns. 1984.6 0.5 .0955

[23] Bland JM, Altman DG. Statistical methods for assessing agreement between two methods of clinical measurement. Lancet. 1986; 1(8476): 307-310. https ://doi .org/10.1016/S0140-6736 (86 ) $90837-8$ 